Research Paper

\title{
Clinical Effect of Adjuvant Cytokine-Induced Killer Cells Immunotherapy in Patients with Stage II-IVB Nasopharyngeal Carcinoma after Chemoradiotherapy: A propensity score analysis
}

Jing-Jing Zhao ${ }^{1,2^{*}}$, Shu Zhou ${ }^{1,3^{*}}$, Chang-Long Chen ${ }^{1,2^{*}}$, Hong-Xia Zhang ${ }^{1}$, Zi-Qi Zhou ${ }^{1,2}$, Zheng-Rong Wu ${ }^{4}$, Yuan Liu ${ }^{1,2}$, Qiu-Zhong Pan ${ }^{1,2}$, Qian Zhu ${ }^{1,2}$, Yan Tang ${ }^{1,2}$, Jian-Chuan Xia ${ }^{1,2}$, De-Sheng Weng ${ }^{1,2}$

1. Collaborative Innovation Center for Cancer Medicine, State Key Laboratory of Oncology in South China, Guangdong Key Laboratory of Nasopharyngeal Carcinoma Diagnosis and Therapy, Sun Yat-sen University Cancer Center, Guangzhou, China

2. Department of Biotherapy, Sun Yat-sen University Cancer Center, Guangzhou, China

3. Department of Radiation Oncology, Sun Yat-sen University Cancer Center, Guangzhou, China

4. Department of Pathology, School of Basic Medicine, Southern Medical University, Guangzhou, China

* These authors contributed equally to this work

$\triangle$ Corresponding author: De-Sheng Weng, PhD, MD. State Key Laboratory of Oncology in South China; Department of Biotherapy, Sun Yat-sen University Cancer Center, 651 Dongfeng Road East, Guangzhou 510060, P. R. China. Tel.: +86-20-87345699, Fax: +86-20-87343392, Email: wengdsh@ sysucc.org.cn Jian-Chuan Xia, PhD, MD. State Key Laboratory of Oncology in South China; Department of Biotherapy, Sun Yat-sen University Cancer Center, 651 Dongfeng Road East, Guangzhou 510060, P. R. China. Tel.: +86-20-87345699, Fax: +86-20-87343392, Email: xiajch@mail.sysu.edu.cn

(C) Ivyspring International Publisher. This is an open access article distributed under the terms of the Creative Commons Attribution (CC BY-NC) license (https://creativecommons.org/licenses/by-nc/4.0/). See http://ivyspring.com/terms for full terms and conditions.

Received: 2018.02.27; Accepted: 2018.07.27; Published: 2018.10.20

\begin{abstract}
As an adjuvant immunotherapy, cytokine-induced killer cells (CIKs) infusion has been demonstrated to exert potent effectiveness in several types of cancer patients who received curative treatment. However, controversy exists regarding whether nasopharyngeal carcinoma (NPC) patients can benefit from additional treatment after radical radiotherapy or chemoradiotherapy to improve their distant control and survival. In this retrospective study, we aimed to evaluate the efficacy of adjuvant CIK cells therapy in NPC patients with stage II-IVB after curative treatment. From January 1, 2005 to December 31, 2012, 85 pairs of NPC patients matching by propensity score matching (PSM) method to balance prognostic factors were included in this study: 85 cases underwent radical treatment, 85 cases received radical treatment and sequential CIKs infusion. We found that disease-free survival (DFS) and overall survival (OS) were significantly better in the CIK group than that in the control group $(P=0.009, P<0.001$, respectively). Adjuvant CIK cells immunotherapy was showed to be an independent prognostic factor for survival of the patients in further multivariate analysis. In subgroup analyses, the DFS and OS of patients with T3/4, III and IV A-B TNM (tumor-node-metastasis) stages were significantly enhanced in CIK group compared to control group. Nevertheless, both NPC patients with high and low EBV DNA benefited from adjuvant CIK cells immunotherapy. In conclusion, CIKs infusion is an effective adjuvant immunotherapy for enhancing the prognosis of NPC patients who have received the standard treatment, particularly for those with more aggressive tumor (T3/4) or advanced TNM stage.
\end{abstract}

Key words: cytokine-induced killer cells; nasopharyngeal carcinoma; disease-free survival; overall survival; clinical effect

\section{Introduction}

Nasopharyngeal carcinoma (NPC), which represents a small proportion of head-and-neck cancers, is one of the most common malignant cancers in south Asia and southern China especially 
Guangdong province [1,2].Although most patients with the disease are diagnosed when the tumor has reached an advanced stage (stages III-IVB) [2], the overall survival rate of them has been significantly improved due to the advances in radiotherapy and the extensive use of chemotherapy [3].However, distant metastasis remains the major cause of treatment failures after the use of radiotherapy and chemotherapy [4]. Nowadays, more potent therapy after the radical treatment is called for improving distant control and prognosis for NPC patients, especially for those with advanced disease [5], whereas the problem whether or not adjuvant treatment should be given stills controversial.

Currently, the approaches to improve disease control and prognosis for cancer patients have concentrated on immunotherapy such as dendritic cell vaccines and adoptive transfer of immune effector cells [6,7]. Emerging studies have proved that cellular immunotherapy can suppress cancer formation and progression in NPC patients by tumor immune response [8-10], indicating that immune-based therapy could be a promising therapy for patients with NPC. As one type of adoptive cellular immunotherapy, transfusion of cytokine-induced killer cells (CIKs) has been used as adjuvant treatment for reducing tumor recurrence and improving survival benefit in several types of solid tumors, such as hepatocellular carcinoma, gastric cancer, renal cell carcinoma, lung cancer, melanoma and triple-negative breast cancer [11-19]. A series of reports have also highlighted the safety and effectiveness of adjuvant CIK treatment in clinical practice [20-24], suggesting that CIK cells represent an effective therapy method in the field of cancer immunotherapy. Previously, we demonstrated that sequential adjuvant CIK enhanced the efficacy of the gemcitabine plus cisplatin chemotherapy egimen for metastatic NPC patients $[25,26]$. However, the clinical effect of adjuvant CIK cells therapy in patients with stage II-IVB remains unclear.

In this study, we retrospectively assessed the clinical efficacy of autologous CIK infusion after the curative treatment including radiotherapy and chemotherapy for the NPC patients with stage II-IVB. Our data provide additional evidence on whether adjuvant CIK immunotherapy could improve the clinical outcomes in patients with NPC.

\section{Materials and Methods}

\section{Patients population}

After obtaining institutional review board approval, the medical records of patients with stage II-IVB NPC were reviewed at Sun Yat-sen University Cancer Center (SYSUCC) between January 1, 2005 and December 31，2012. These records included the clinicopathological information of the patients at accrual such as age, sex, TNM (tumor-nodemetastasis) stage, treatment and outcome. NPC was histologically diagnosed based on the World Health Organization criteria. All patients in this study had adequate baseline cardio-pulmonary and renal function, and the Eastern Cooperative Oncology Group performance status score were $\leq 2$. The following exclusion criteria were applied: with relapse or distant metastasis, with autoimmune disease or primary immunodeficiency, with serious adverse events during previous treatment, a history of other malignancy, or recruitment in other clinical trial. After review, 8753 NPC patients received either adjuvant CIK treatment or not after radical treatment were included. Propensity score matching (PSM) was then used to match the patients who received CIK treatment and the patients who did not receive. Finally, 85 patients in CIK treatment group and 85 patients in control group. All covariates between the two groups achieved an adequate balance by the PSM (Table 1).

Table 1. Distribution of demographic and clinical characteristics of patients in the control and CIK treatment groups

\begin{tabular}{|c|c|c|c|c|}
\hline Characteristic & All Patients $n=170$ & Control group $n=85$ & CIK group $n=85$ & $P_{c}$ \\
\hline Age, No. (\%) & & & & 0.514 \\
\hline$<50 y$ & $114(67.1)$ & $55(64.7)$ & $59(69.4)$ & \\
\hline$\geq 50 \mathrm{y}$ & $56(32.9)$ & 30(35.3) & $26(30.6)$ & \\
\hline Gender, No. (\%) & & & & 0.323 \\
\hline Male & $116(68.2)$ & $55(64.7)$ & 61(71.8) & \\
\hline Female & $54(31.8)$ & 30(35.3) & $24(28.2)$ & \\
\hline WHO Histologya, No. (\%) & & & & 0.650 \\
\hline II & $5(2.9)$ & $3(3.5)$ & $2(2.4)$ & \\
\hline III & 165(97.1) & $82(96.5)$ & 83(97.6) & \\
\hline EBV DNA, No. (\%) & & & & 0.539 \\
\hline$<4000$ copies $/ \mathrm{mL}$ & $88(51.8)$ & $42(49.4)$ & $46(54.1)$ & \\
\hline$\geq 4000$ copies $/ \mathrm{mL}$ & $82(48.2)$ & $43(50.6)$ & $39(45.9)$ & \\
\hline T stage, No. (\%) & & & & 0.866 \\
\hline T1 & $14(8.2)$ & 6(7.1) & $8(9.4)$ & \\
\hline
\end{tabular}




\begin{tabular}{|c|c|c|c|c|}
\hline $\mathrm{T} 2$ & $47(27.6)$ & $22(25.9)$ & $25(29.4)$ & \\
\hline $\mathrm{T} 3$ & $81(47.7)$ & $42(49.4)$ & $39(45.9)$ & \\
\hline $\mathrm{T} 4$ & $28(16.5)$ & 15(17.6) & $13(15.3)$ & \\
\hline N stage, No. (\%) & & & & 0.829 \\
\hline No & $26(15.3)$ & $12(14.1)$ & $14(9.5)$ & \\
\hline N1 & $70(41.2)$ & $38(44.7)$ & $32(37.6)$ & \\
\hline N2 & $55(32.3)$ & $26(30.6)$ & $29(34.1)$ & \\
\hline N3 & 19(11.2) & $9(10.6)$ & $10(11.8)$ & \\
\hline TNM stage ${ }^{b}$, No. $(\%)$ & & & & 0.932 \\
\hline II & $39(22.9)$ & $19(22.4)$ & $20(23.5)$ & \\
\hline III & $93(54.7)$ & $46(54.1)$ & $47(55.3)$ & \\
\hline IVa-b & $38(22.4)$ & $20(23.5)$ & $18(21.2)$ & \\
\hline Chemotherapy, No. (\%) & & & & 0.868 \\
\hline IC & $28(16.5)$ & $15(17.6)$ & $13(15.3)$ & \\
\hline $\mathrm{CC}$ & $50(29.4)$ & $25(29.4)$ & $25(29.4)$ & \\
\hline $\mathrm{IC}+\mathrm{CC}$ & $61(35.9)$ & $29(34.1)$ & $32(37.6)$ & \\
\hline Radiotherapy, No. (\%) & & & & 0.707 \\
\hline IMRT & $134(78.8)$ & $66(77.6)$ & $68(80.0)$ & \\
\hline Other & $36(21.2)$ & $19(22.4)$ & $17(20.0)$ & \\
\hline
\end{tabular}

Abbreviations: $\mathrm{CIKs}=$ cytokine-induced killer cells, IC $=$ induction chemotherapy, $\mathrm{CC}=$ concurrent chemotherapy IMRT= intensity-modulated radiotherapy . ${ }^{\mathrm{a}} \mathrm{Based}$ on the criteria of WHO histological type (1991): II-Differentiated non-keratinising carcinoma, III-Undifferentiated non-keratinising carcinoma. ${ }^{\mathrm{b}}$ According to the 7 th edition of the International Union against Cancer/American Joint Committee on Cancer staging system. ${ }^{c} P$-values were calculated using $X^{2}$ test.

\section{Treatment protocol}

All NPC patients were treated with the either radical radiotherapy or with chemoradiotherapy. The overall median radiotherapy dose was $69.7 \mathrm{~Gy}$ (range, 68-70), the overall median dose per fraction was 2.19 Gy (range, 2.12-2.26) and the overall median duration of radiotherapy was 45 days (range, 42-49). Induction chemotherapy (IC) was administered to the patients as follows: PF regimen (cisplatin and 5-Fu), TPF (docetaxel, cisplatin, and 5-Fu). Cisplatin regimen was used for concurrent chemoradiotherapy (CCRT). One month after completion of radiotherapy or CCRT, CIK group patients received immune cell infusion. The cell preparation and infusion protocol are described below.

\section{CIKs generation}

The preparation of autologous CIK cells has been described in our previous reports $[13,15,18]$. Briefly, when routine blood examination returned to normal, $50-60 \mathrm{ml}$ of heparinized peripheral blood was collected from each patient who completed the radiotherapy or CCRT treatment. Peripheral blood mononuclear cells (PBMCs) were isolated using Ficoll-Hypaque gradient centrifugation and cultured in X-VIVO 15 serum-free medium. CIK cells were generated using $1000 \mathrm{U} / \mathrm{ml}$ rhIFN- $\gamma$ for the first day followed by stimulation with $100 \mathrm{ng} / \mathrm{ml}$ OKT-3, 1000 $\mathrm{U} / \mathrm{ml}$ rhIL-2 and $100 \mathrm{U} / \mathrm{ml}$ IL-1a. Fresh medium containing 1,000 U/mL rhIL-2 was supplemented periodically during the culture. At 14 days, the CIK cells were harvested, and their number, viability, and whether they were contaminated were evaluated.

\section{CIKs treatment}

The treatment of CIK cells infusion is an observational clinical immunotherapy in SYSUCC.
The CIKs treatment was approved by the institutional ethics committee of SYSUCC. Written informed consent was obtained from each patient before treatment. After being cultured for 14 days, all numbers of harvested autologous CIK cells free of microbial contamination were washed and resuspended with $100 \mathrm{~mL}$ normal saline supplemented with $1 \%$ human serum albumin. The fresh CIK cells were administered via intravenous infusion within 30 minutes. Before infusion, 50 to 60 $\mathrm{mL}$ heparinized peripheral blood was collected for the next cycle of CIK generation. Treatment-related adverse events (AEs) such as fever, fatigue, chills, and vomiting were monitored and recorded during the treatment and observation period. Generally, patients received at least four cycles of CIK cell treatment at two-week intervals. The details of CIK cell treatment assignment procedure was showed in Fig. S1. If the patients were disease-stable, they were eligible for more cycles of CIK maintenance treatment using the above protocol. Otherwise, CIK therapy was stopped once the disease is progression or the patients did not want to continue.

\section{Follow-up}

All patients in this study had undergone detailed follow-up at our outpatient department after radiotherapy or CCRT. In general, patients were contacted every 3 months for the first 2 years, every 6 months for years 3-5, and annually thereafter or until the patient's death, whichever occurred first. Clinical and laboratory examinations were obtained at each follow-up visit. When tumor recurrence was suspected, additional evaluations such as pulmonary and abdominal CT, nasopharyngeal and cervical MRI as well as ECT scans of whole-body bone were performed. Disease-free survival (DFS) was calculated 
from the time of initial radiotherapy or CCRT to the time of first failure (local or distant) or until the last follow-up. Overall survival (OS) was defined from the date of the initial radiotherapy or CCRT to the date of death from any cause or the last follow-up. If recurrence or metastasis were confirmed during the follow-up, the patients received multidisciplinary synthetic therapy according to the recommendation by physicians. The statues and correlating treatment of patients were entered into medical records after follow-up and updated accordingly in the database.

\section{Flow cytometry analysis}

The phenotype of the autologous CIK cells was characterized by flow cytometry. CIK cells were resuspended at $2 \times 10^{5}$ cells per $100 \mu \mathrm{L}$ of phosphate-buffered saline (PBS) and incubated for 30 min at $4^{\circ} \mathrm{C}$ with the following anti-human antibodies: anti-CD3-PE-Cy5, anti-CD4-FITC, anti-CD8-PE-CF594 and anti-CD56-PE-Cy7 (all from BD Bioscicence). The cells were analyzed using a CytomicsTM FC500 Flow Cytometer (Beckman Coulter, USA). Data analysis was performed with CXP analysis software (Beckman Coulter, USA).

\section{Cytotoxicity assays and cell lines culture}

The cytotoxic specificity of the CIK cells obtained from five patients in the CIK group was analyzed using a Cyto Tox 96 Lactate Dehydrogenase Assay Kit (Promega) according to the manufacturer's protocol. The effector cells in these tests were CIK cells and the target cells were NPC cell lines S18 and 5-8F which were obtained from the Committee of the Type Culture Collection of the Chinese Academy of Sciences (Shanghai, China) and cultured at $37^{\circ} \mathrm{C}$ in $5 \%$ $\mathrm{CO} 2$ in DMEM medium (Gibco, USA) supplemented with $10 \%$ fetal bovine serum (FBS; Gibco, USA) and $1 \%$ penicillin-streptomycin. Cytotoxicity was quantified after the effector and target cells were co-incubated for $12 \mathrm{~h}$ at an effector cell to target cell (E: T) ratio of 3:1, 10:1, or 30:1.

\section{Statistical analysis}

All statistical analyses were conducted using SPSS version 20.0 (SPSS, Inc., Chicago, IL, USA). PSM was computed by logistic regression for each patient using the following covariates: age, gender, WHO Histology, smoking, EBV DNA, T stage, N stage, TNM stage, chemotherapy, and radiotherapy. Pearson $\chi 2$ test and the Fisher's exact test were used to analyze differences in demographic and clinical variables of the two groups. DFS and OS curves were evaluated by the Kaplan-Meier method and compared by the log-rank test. Univariate and multivariate regression analyses were performed using a Cox regression hazard model. A difference of $p<0.05$ was considered statistically significant in all tests.

\section{Results}

\section{Patient characteristics}

After matching by PSM, A total of 85 pairs were included in the present retrospective analysis. Among them, $116(68.2 \%)$ were men and $54(31.8 \%)$ were women, and the median age was 44 years old (range, $15-76$ years old). 134 patients $(n=66$ in control group; $n=68$ in CIK group) received intensity-modulated radiotherapy (IMRT) and the other 36 patients $(n=19$ in control group; $n=17$ in CIK group) received conventional radiotherapy. 45 patients in the CIK group and 44 patients in the control group received 3 cycles IC followed by radiotherapy or CCRT. The clinicopathological parameters between CIK and control groups were well matched (Table 1). No statistically significant differences between the two groups were found in terms of all variables including age, gender, pathological category (WHO), EBV-DNA, T, N, TNM stage and previous radiotherapy and chemoradiotherapy (Table $1, P>$ 0.05).

\section{Characteristics of the cultured CIK cells}

The total number of cultured CIK cells at the time of transfusion was an average of $10 \times 10^{9} \mathrm{cells}$ (range, 8.7-15 $\times 10^{9}$ ). The viability of the immune cells was over $95 \%$ with free of bacterial or fungal contamination, negative for mycoplasma and contained endotoxin $<5 \mathrm{EU}$. The infused CIK cells were prominently CD3+T cells (median, $93.9 \%$; range, $78.2 \%$ to $99.1 \%$ ), comprising $\mathrm{CD}^{+} \mathrm{CD}^{+} \mathrm{T}$ cells (median, 60.5\%; range,41.1\%-80.2\%), $\mathrm{CD}^{+}{ }^{+} \mathrm{CD} 4{ }^{+} \mathrm{T}$ cells (median, 30.2\%; range, $18.3 \%$ to $44.2 \%$ ), CD3-CD56 ${ }^{+} \mathrm{NK}$ cells (median, $3.4 \%$; range, $1.3 \%$ to $21.6 \%$ ), and $\mathrm{CD}^{+}{ }^{+} \mathrm{CD} 56^{+} \mathrm{NKT}$ cells(median, $15.8 \%$; range, $8.4 \%-34.7 \%)$. After evaluation, all fresh autologous CIK cells were transfused into the patients. CIK cells phenotype before and after culture from one of the study patients were shown in Fig. 1A-D. We also chose five patients in the CIK group patients to evaluate the cytolytic activity of the CIK cells. CIK cells were co-cultured with S18 and 5-8F cells at a 3:1, 10:1, or 30:1 CIK to tumor cell ratio (E: T ratio). As shown in Fig. 1E, for the S18 cell line, the lysis ratio of the CIK cells was $13.36 \pm 2.19 \%$ at $3: 1 \mathrm{E}: \mathrm{T}$ ratio, $46.66 \pm 9.60 \%$ at $10: 1 \mathrm{E}$ : $\mathrm{T}$ ratio and $83.75 \pm 5.19 \%$ at 30:1 E: T ratio, respectively (Fig. 1E). For the $5-8 \mathrm{~F}$ cell line, the lysis ratio of the CIK cells was $15.43 \pm$ $2.99 \%$ at $3: 1 \mathrm{E}$ : $\mathrm{T}$ ratio, $43.49 \pm 7.39 \%$ at $10: 1 \mathrm{E}$ : $\mathrm{T}$ ratio and $74.29 \pm 4.09 \%$ at $30: 1 \mathrm{E}: \mathrm{T}$ ratio, respectively (Fig. 1E). 


\section{Treatment-related AEs of CIK cell immunotherapy}

AEs were evaluated by the protocol with National Cancer Institute Common Toxicity Criteria version 4.0, which were defined drug related or that occurred in patients during medical treatment or procedure regardless of relationship to drug. The treatment-related AEs of CIK cells treatment include fever, fatigue, chills, vomiting, anemia, leucopenia and autoimmune disorder. Among the CIK group, AEs occurred in 14 cases (16.5\%) during they received CIK cells infusion, 10 of which were fever, chills, and fatigue, at grade 1 or 2, and spontaneously resolved within $12 \mathrm{~h}$. Another four patients appeared leucopenia and recovered by symptomatic treatment. No infections, vomiting, allergic reactions or autoimmune disorder were observed following infusion with CIK cells. No treatment-related serious AEs such as pneumonitis and treatment-related

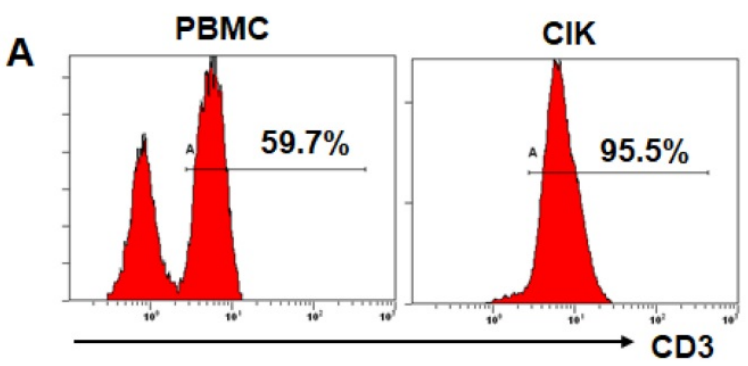

C
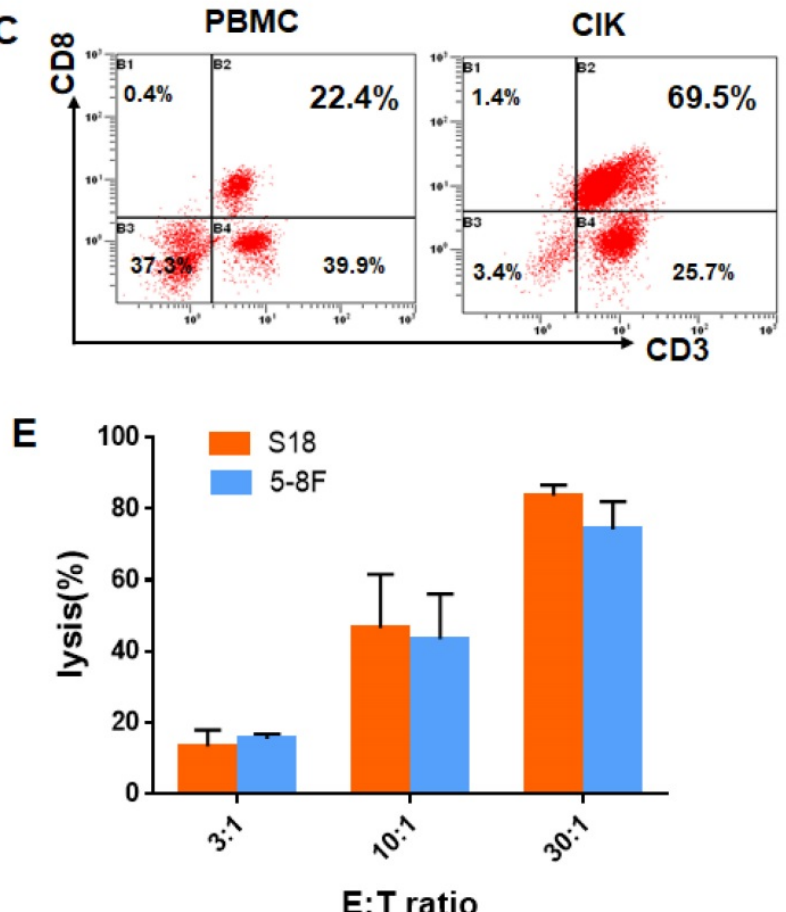

deaths appeared in any of the patients.

\section{Prognosis of patients in the two groups}

The median follow-up period for all patients was 78.3 months (range, 7.4-132.5 months). DFS and OS rates at 1,3 , and 5 years after curative radiotherapy or chemoradiotherapy were $94.1 \% \quad(99.4 \%), \quad 74.3 \%$ $(89.0 \%)$, and $64.0 \%$ (78.2\%) for the whole study population. Survival analysis showed that patients in the CIK group had a significantly enhanced DFS and OS compared to those in the control group (Fig. 2A and 2B). The median DFS and OS time were 76.6 and 81.2 months for patients in the CIK group compared to 61.3 and 68.6 months for patients in the control group, respectively. The 1-, 3-, and 5-year DFS rates were $95.3 \%, 81.1 \%$, and $72.3 \%$, respectively, for the CIK group compared with $92.9 \%, 67.4 \%$, and $55.6 \%$, respectively, for the control group (log-rank test, $P=$ 0.009 ). The 1-, 3-, and 5-year OS rates were $100 \%$, $95.2 \%$, and $88.9 \%$, respectively, for the CIK group
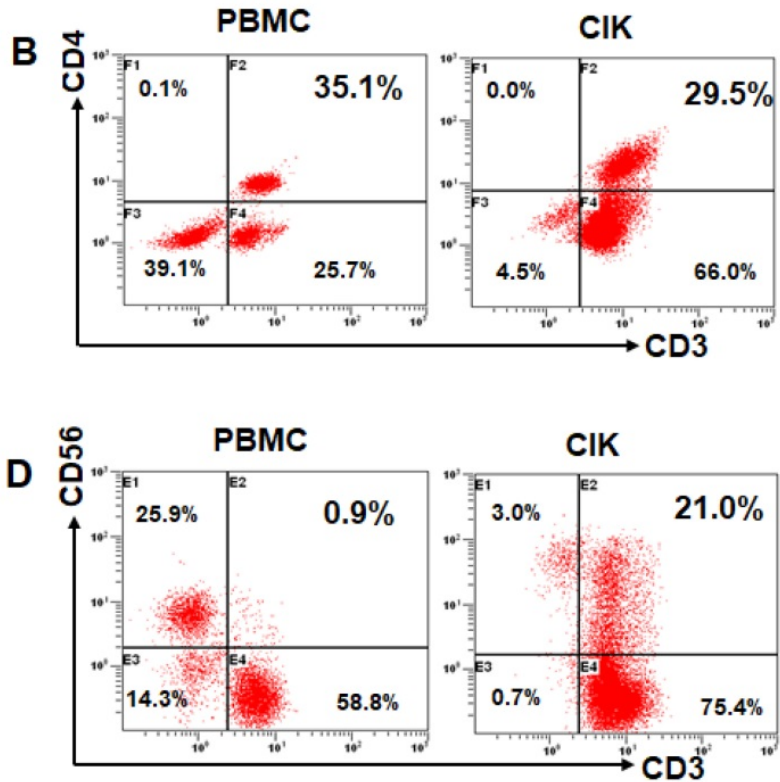

Figure 1. CIK cells phenotype before and after culture from one of CIK group patients. (A) The percentage of CD3+T cells of the PBMC and CIK cells. (B) The percentage of $C D 3^{+} C D 4+T$ cells of the PBMC and CIK cells. (C) The percentage of CD3+CD8+T cells of the PBMC and CIK cells. (D) The percentage of $\mathrm{CD}^{+}{ }^{+} \mathrm{CD} 56^{+} \mathrm{NKT}$ cells of the PBMC and CIK cells. (E) The cytolytic activity of CIK cells in response to two NPC cell lines, S18 and 5-8F, at a 3:1, 10:1, or 30:1 $\mathrm{E}: \mathrm{T}$ ratio. $\mathrm{E}: \mathrm{T}$ ratio, effector cell to target cell ratio. 
compared with $98.8 \%, 82.8 \%$, and $67.4 \%$, respectively, for the control group (log-rank test, $P<0.001$ ). The effects of CIKs treatment on the prognosis of NPC patients with curative radiotherapy or chemoradiotherapy were further evaluated by univariate and multivariate Cox proportional hazards regression analyses. Low EBV DNA, early T stage, early TNM

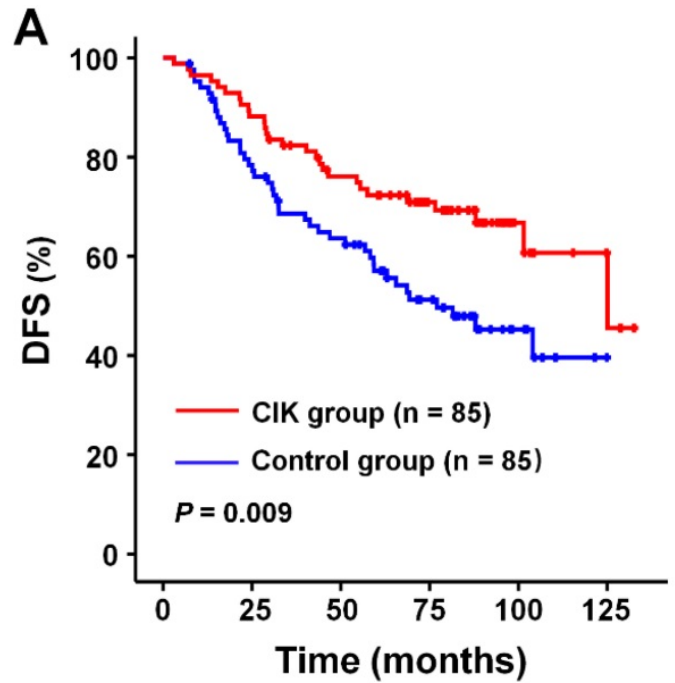

stage, and adjuvant CIKs treatment were significantly associated with better DFS and OS in univariate analysis (Table 2 and Table 3). Multivariate survival analysis showed that Low EBV DNA, early TNM stage, and adjuvant CIK treatment were the independent prognostic factor for improved DFS and OS (Table 2 and Table 3 ).

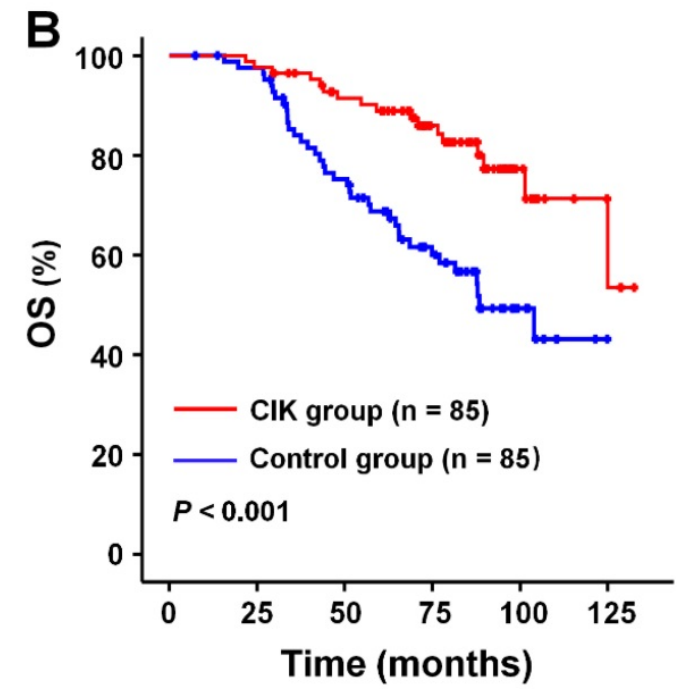

Figure 2. Survival analysis in patients with nasopharyngeal carcinoma. Kaplan-Meier method was used to compare the survival rates between the sequential CIK adjuvant therapy group (CIK group, $n=85$ ) and radiotherapy with/without chemotherapy treatment alone group (control group, $n=85$ ). (A) DFS curves. (B) OS curves. Significantly improved DFS and OS were observed in the CIK group versus the control group. $P$ values were calculated using the log-rank test.

Table 2. Univariate and multivariate analysis of disease-free survival in patients with nasopharyngeal carcinoma

\begin{tabular}{|c|c|c|c|c|c|c|}
\hline \multirow[t]{2}{*}{ Variables } & \multicolumn{3}{|c|}{ Univariate analysis } & \multicolumn{3}{|c|}{ Multivariate analysis } \\
\hline & $\mathrm{HR}$ & $95 \% \mathrm{CI}$ & $P$ & HR & $95 \% \mathrm{CI}$ & $P$ \\
\hline Age (<50 vs. $\geq 50)$ & 1.078 & $0.662-1.754$ & 0.764 & & & \\
\hline Gender (male vs. female) & 0.737 & $0.435-1.249$ & 0.257 & & & \\
\hline WHO Histology (II vs. III) & 2.024 & $0.281-14.595$ & 0.484 & & & \\
\hline EBV DNA (< 4000 vs. $\geq 4000)$ & 2.187 & $1.341-3.566$ & $0.002^{\mathrm{a}}$ & 2.152 & $1.317-3.516$ & $0.002^{\mathrm{a}}$ \\
\hline T stage $(1,2$ vs. 3,4$)$ & 1.841 & $1.085-3.123$ & $0.024^{\mathrm{a}}$ & 1.279 & $0.742-2.216$ & 0.396 \\
\hline $\mathrm{N}$ stage (0 vs. $1,2,3)$ & 1.338 & $0.685-2.613$ & 0.395 & & & \\
\hline TNM stage (II vs. III vs. IVa-b) & 1.973 & $1.370-2.841$ & $<0.001^{\mathrm{a}}$ & 1.847 & $1.244-2.742$ & $0.002^{\mathrm{a}}$ \\
\hline Treatment (CIK vs. control) & 0.533 & $0.329-0.864$ & $<0.001^{\mathrm{a}}$ & 0.543 & $0.335-0.882$ & $0.014^{\mathrm{a}}$ \\
\hline
\end{tabular}

$H R$ Hazard ratio, $C I$ confidence interval

a $P$ value $<0.05$

Table 3. Univariate and multivariate analysis of overall survival in patients with nasopharyngeal carcinoma

\begin{tabular}{|c|c|c|c|c|c|c|}
\hline \multirow[t]{2}{*}{ Variables } & \multicolumn{3}{|c|}{ Univariate analysis } & \multicolumn{3}{|c|}{ Multivariate analysis } \\
\hline & $\mathrm{HR}$ & $95 \% \mathrm{CI}$ & $P$ & HR & $95 \% \mathrm{CI}$ & $P$ \\
\hline Age (<50 vs. $\geq 50)$ & 1.542 & $0.896-2.656$ & 1.542 & & & \\
\hline Gender (male vs. female) & 0.842 & $0.463-1.533$ & 0.574 & & & \\
\hline WHO Histology (II vs. III) & 1.172 & $0.162-8.499$ & 0.875 & & & \\
\hline EBV DNA $(<4000$ vs. $\geq 4000)$ & 2.246 & $1.274-3.962$ & $0.005^{\mathrm{a}}$ & 2.261 & $2.276-4.007$ & $0.005^{\mathrm{a}}$ \\
\hline T stage $(1,2$ vs. 3,4$)$ & 2.771 & $1.422-5.400$ & $0.003^{\mathrm{a}}$ & 1.785 & $0.879-3.625$ & 0.109 \\
\hline $\mathrm{N}$ stage (0 vs. $1,2,3)$ & 1.279 & $0.602-2.718$ & 0.522 & & & \\
\hline TNM stage (II vs. III vs. IVa-b) & 2.452 & $1.598-3.762$ & $<0.001^{\mathrm{a}}$ & 2.197 & $1.368-3.531$ & $0.001^{\mathrm{a}}$ \\
\hline Treatment (CIK vs. control) & 0.350 & $0.194-0.629$ & $<0.001^{a}$ & 0.358 & $0.198-0.647$ & $0.001^{\mathrm{a}}$ \\
\hline
\end{tabular}

HR Hazard ratio, CI confidence interval

a $P$ value $<0.05$ 


\section{Subgroup analysis for the NPC patients benefit from adjuvant CIK treatment}

We subsequently identify subgroup of the NPC patients who could benefit more from adjuvant CIK cells treatment. Because EBV DNA, T stage, and TNM stage were related to the prognosis of the patients, we evaluated the survival benefit from the CIK cells treatment based on these clinical parameters. In the early stage subgroup (T1/2 and II TNM stages), the DFS and OS of patients with NPC did not significantly benefit from CIK treatment (Fig. 3A and 4A). In the advanced stage subgroup (T3/4 and III, IVa-b TNM stages), the DFS and OS of patients with NPC were significantly enhanced in the CIK group compared to the control group (Fig. 3B, 4B and 4C). We found that the EBV DNA level had no statistically significant difference between the control and CIK groups in the T1/2, T3/4, II, III and IV subgroups, respectively (Table S1, $P=0.363 ; P=0.899 ; P=0.621 ; P=0.598 ; P=$ 0.098 , respectively). Furthermore, between the T1/2 and T3/4 subgroups the EBV DNA level of the NPC patients had no significant difference, as well as that among the II, III and IV subgroups (Table S2, $P=$ $0.614 ; P=0.722$, respectively). However, both in the low and in the high EBV DNA subgroup, the NPC patients who received curative radiotherapy or chemoradiotherapy could benefit from the adjuvant CIK cell immunotherapy (Fig. 5A and 5B). The distribution of clinical stage of the NPC patients was uniform between the EBV high and low groups (Table S3, $P=0.722)$.
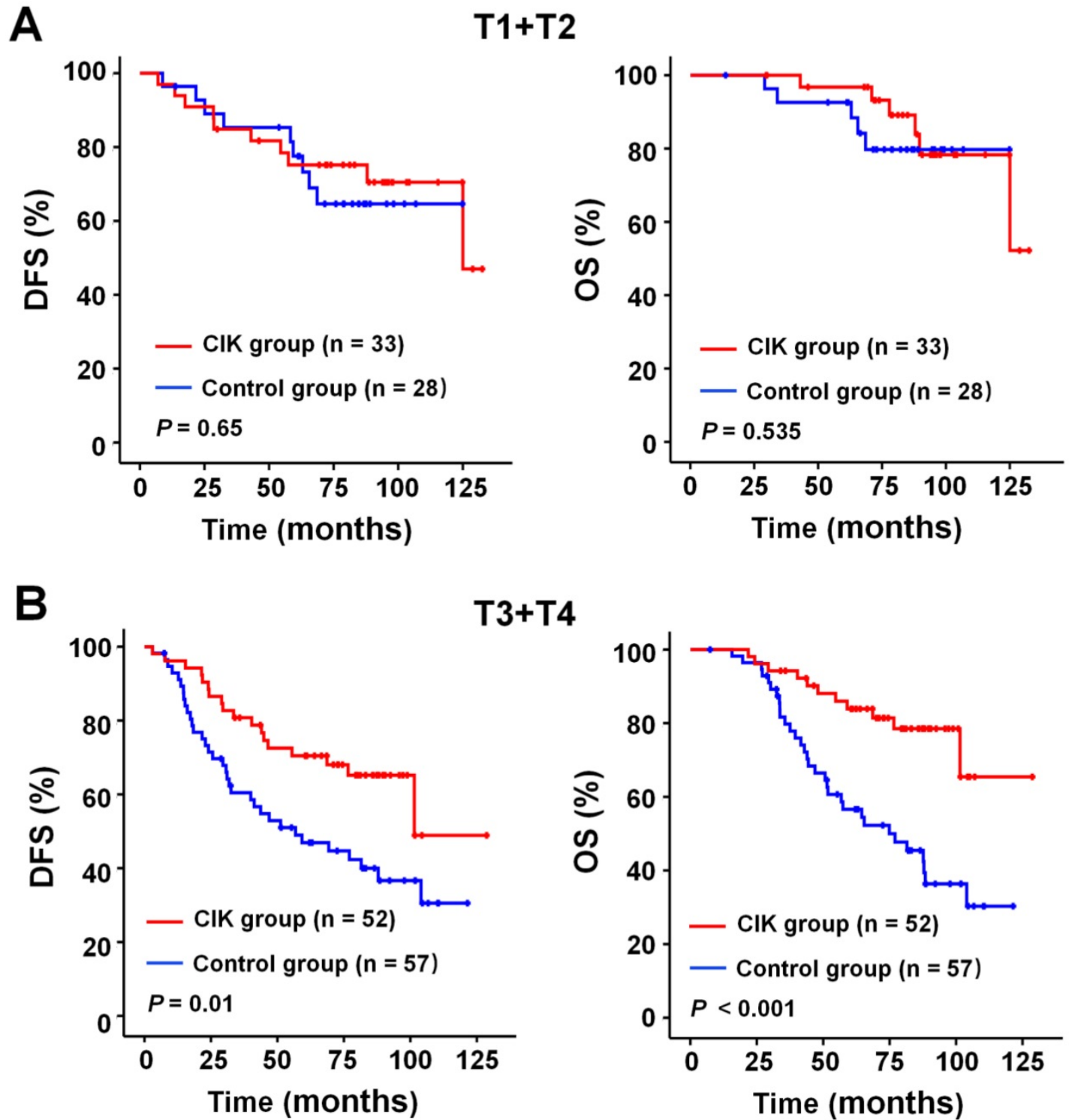

$\mathrm{T} 3+\mathrm{T} 4$

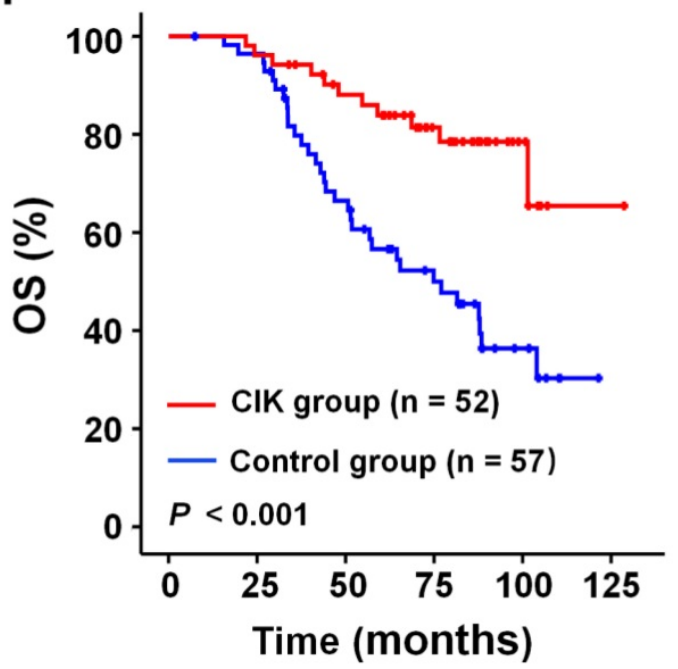

Figure 3. Subgroup analysis to evaluate the benefits of adjuvant CIK treatment according to $T$ stage. (A) DFS and OS curves for patients with early $T$ stage (T1/2). (B) DFS and OS curves for patients with advanced T stage (T3/4). 

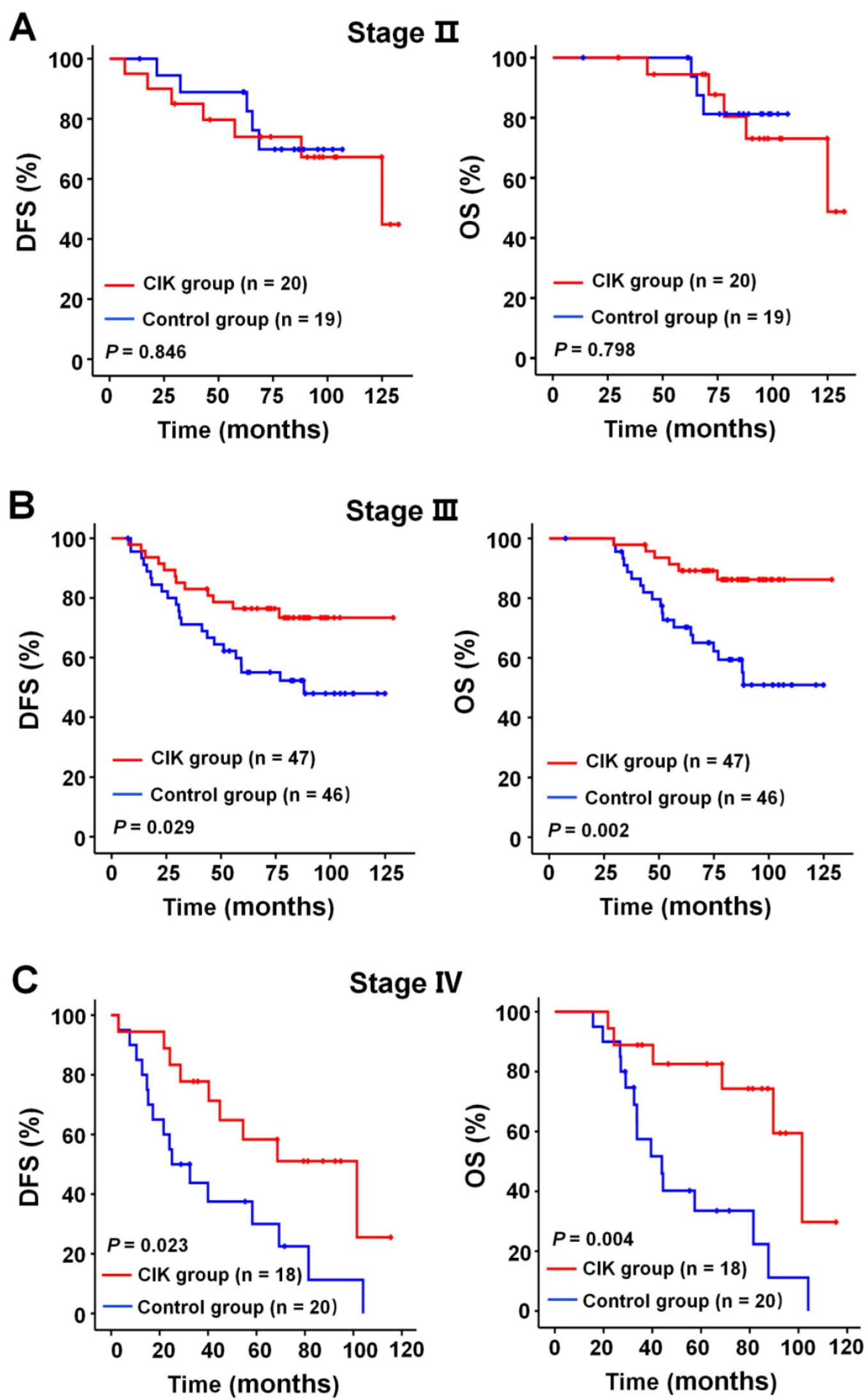

Figure 4. Subgroup analysis to estimate the benefits of adjuvant CIK treatment according to TNM stage. (A) DFS and OS curves for patients with II TNM stage. (B) DFS and OS curves for patients with III TNM stage. (C) DFS and OS curves for patients with IV TNM stage. 

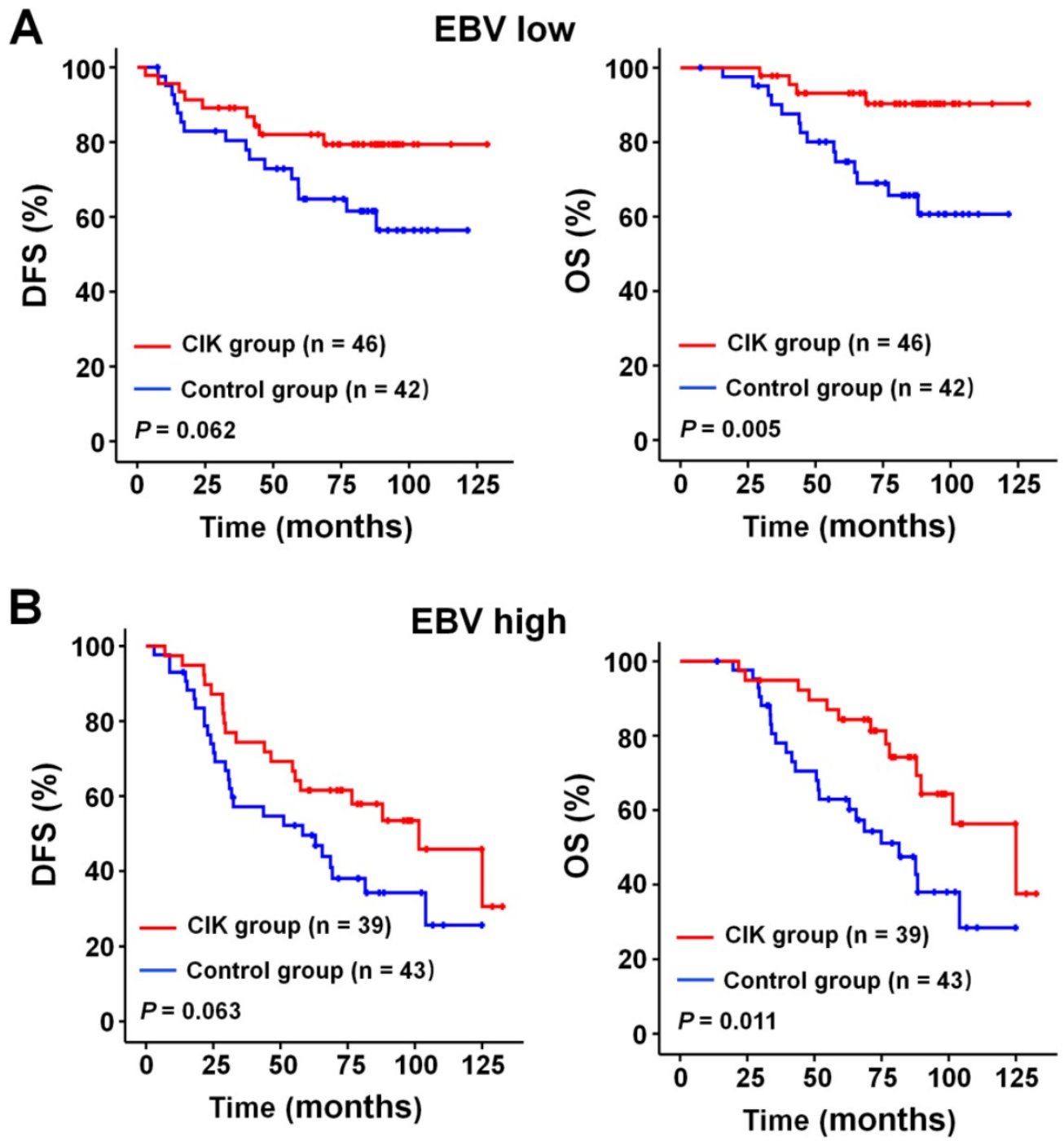

Figure 5. Subgroup analysis to evaluate the benefits of adjuvant CIK treatment based on EBV DNA load. (A) DFS and OS curves for patients with low EBV DNA load. (B) DFS and OS curves for patients with high EBV DNA load.

\section{Discussion}

Avoiding immune destruction has been considered an emerging hallmark of cancer, suggesting the host immune system plays a key role as a barrier to tumor formation and progression [27]. In the past few years, a lot of clinical studies have demonstrated that CIKs treatment show positive clinical efficacy in several types of cancer $[13-15,18,19]$. Indeed, to date, previous studies have increasingly confirmed the therapeutic effects of CIKs treatment in patients with relapse and metastatic NPC $[10,25,26]$. In the present study, we retrospectively evaluated the efficacy of sequential CIK infusion in stage II-IVB NPC patients who have received curative radiotherapy or chemoradiotherapy.

With the analysis of 170 patients, we found that, compared to the control group that received only curative radiotherapy or chemoradiotherapy, patients with NPC who received adjuvant sequential CIKs treatment significantly improved survival, including DFS and OS. This result is similar to the findings of $\mathrm{Li}$ et al [25]. Furthermore, multivariate survival analysis showed that the CIKs treatment was an independent prognostic factor for the patients' survival. All these results indicated that CIK treatment is an effective intervention that prolongs the survival of patients with NPC. Our conclusions are in accordance with previous studies in patients with lung cancer $[15,28]$, gastric cancer [16], triple-negative breast cancer [13], colorectal cancer [29], liver cancer [14,18] who received adjuvant CIKs treatment. The retrospective clinical analyses provided convincing evidence in support of the efficacy of CIK cell-based adjuvant treatment combined with surgery or chemoradiotherapy for improved outcomes of patients with cancer.

It can be speculated that the mechanism which CIK immunotherapy enhance the therapeutic efficacy 
of chemoradiotherapy is based on their synergistic effect. Firstly, CIK cells can enhance the efficacy of chemoradiotherapy in patients by eliminating potential or residual tumor cells including even drug-resistant tumor cells [30-32]. Furthermore, there are reports showed that CIK cells also have intense tumor killing activity in vitro and in vivo against putative cancer stem cells $[33,34]$. It has reported that CIK cells can efficiently kill stem-like cancer cells of nasopharyngeal carcinoma via the NKG2D-ligands recognition [35]. Thirdly, many of the available anticancer drugs can increase the susceptibility of malignant cells to the cytotoxic activity of immune effector cells, which would favor the antitumor functions of immune effector cells [36]. Meanwhile, infused CIK cells can alleviate immune damage and enhance the immunosurveillance capability of NPC patients who have undergone chemoradiotherapy via producing large number of inflammatory cytokines such as IL-2, IFN- $\gamma$, and TNF- $\alpha[37,38]$. On the other hand, chemotherapy can remove immune suppressor factors such as regulatory $\mathrm{T}$ cells and myeloid-derived suppressor cells (MDSCs), which would favor the antitumor functions of immune effector cells [39]. Thus, these findings indicated that conventional chemoradiotherapy in combination with CIK immunotherapy represents an optimization strategy to gain improved therapeutic efficacy in patients with cancer, including patients with NPC.

NPC is an Epstein-Barr virus (EBV)-related malignancy expressing EBV antigens that are possible targets of cell therapy, including latent membrane protein 2 (LPM2). There have been several studies focused on EBV-targeted cell therapy with autologous virus-specific cytotoxic $\mathrm{T}$ lymphocytes (CTLs) transfusion for refractory NPC [40,41]. The results indicated that EBV-specific CTLs are safe and exerting specific killing of NPC tumor cells in vivo [40,41]. A recent phase II study by Chia et al found that gemcitabine and carboplatin chemotherapy followed by EBV-specific CTLs transfusion achieves better survival outcome in NPC patients with metastasis and/or local recurrence [42]. This finding was consistent with our results. Although compared to the EBV-specific CTLs, CIK cells are not antigen specific T cells, CIK cells have several advantages. First, they are easy to culture and produce. With a simple cytokine cocktail, approximately $10^{10}$ cells can be obtained within 2 weeks from an initial culture of $10^{7}$ cells. Second, these cells possess strong antitumor activity and target a broad spectrum of tumors without MHC restriction. Third, minimal toxicity and no graftversus-host disease are found when using allogeneic CIK cells for infusion. These might explain why patients either with low or with high EBV DNA could obtain clinical benefit from adjuvant CIK cell immunotherapy in the present study. To further improve the clinical application of CIK immunotherapy, the potential factors that could have had an impact on the outcome of treatment were also evaluated in our study. In subgroup analyses, adjuvant CIK treatment can significantly improve the prognosis of NPC patients with T3/4 stage or III, IVa-b TNM stage, but not in the patients with T1/2 stage or II TNM stage. As there are often limited therapeutic strategies for patients with advanced cancer, these results indicated that adjuvant CIK immunotherapy provides an effective treatment option for NPC patients with advanced clinical stages.

In conclusion, this retrospective study showed that adjuvant CIK cells treatment after curative radiotherapy or chemoradiotherapy exhibit a better survival improvement for stage II-IVB NPC patients than control group. Furthermore, our results showed that patients with advanced clinical stages might benefit more from adjuvant CIK immunotherapy. Prospective randomized studies are warranted to confirm the present findings and to further define optimal combinational treatment strategies for immunotherapy of NPC.

\section{Supplementary Material}

Supplementary figures and tables.

http://www.jcancer.org/v09p4204s1.pdf

\section{Acknowledgements}

This work was supported by grants from the National Natural Science Foundation of China (No. 81402560; 81472387) and the Guangdong Province Science and Technology Plan Project (No. 2017A020215029; 2014A020212584; 2013B021800063).

\section{Financial disclosure}

All authors have declared there are no financial conflicts of interest in regards to this work.

\section{Authenticity of this article}

Our study has been validated by uploading the key raw data onto the Research Data Deposit public platform (www.researchdata.org.cn) at Sun Yat-Sen University Cancer Center (approval number: RDDB2018000339).

\section{Conflict of interest}

All authors have declared that they have no conflicts of interest regarding this work.

\section{References}

1. Ma BB, Chan AT. Recent perspectives in the role of chemotherapy in the management of advanced nasopharyngeal carcinoma. Cancer. 2005; 103:22-31.

2. Wei WI, Sham JS. Nasopharyngeal carcinoma. Lancet. 2005; 365:2041-54. 
3. Chua MLK, Wee JTS, Hui EP, et al. Nasopharyngeal carcinoma. Lancet. 2015; 387:1012-24

4. Au KH, Ngan RKC, Ng AWY, et al. Treatment outcomes of nasopharyngeal carcinoma in modern era after intensity modulated radiotherapy (IMRT) in Hong Kong: A report of 3328 patients (HKNPCSG 1301 study). Oral Oncol. 2018;77:16-21.

5. Lee AWM, Tung SY, Ng WT, et al. A multicenter, phase 3, randomized trial of concurrent chemoradiotherapy plus adjuvant chemotherapy versus radiotherapy alone in patients with regionally advanced nasopharyngeal carcinoma: 10-year outcomes for efficacy and toxicity. Cancer. 2017; 123:4147-4157.

6. Dudley ME, Rosenberg SA. Adoptive-cell-transfer therapy for the treatment of patients with cancer. Nat Rev Cancer. 2003; 3:666-75.

7. Finn OJ. Cancer vaccines: between the idea and the reality. Nat Rev Immunol. 2003; 3:630-41.

8. Lutzky VP, Crooks P, Morrison L, et al. Cytotoxic $\mathrm{T}$ cell adoptive immunotherapy as a treatment for nasopharyngeal carcinoma. Clin Vaccine Immunol. 2014; 21:256-9.

9. Shengjun W, Yunbo G, Liyan S, et al. Quantitative study of cytotoxic T-lymphocyte immunotherapy for nasopharyngeal carcinoma. Theor Biol Med Model. 2012; 9:6-19.

10. Wei F, Rong XX, Xie RY, et al. Cytokine-induced killer cells efficiently kill stem-like cancer cells of nasopharyngeal carcinoma via the NKG2D-ligands recognition. Oncotarget. 2015; 6:35023-39.

11. Li R, Wang C, Liu L, et al. Autologous cytokine-induced killer cell immunotherapy in lung cancer: a phase II clinical study. Cancer Immunol Immunother. 2012; 61:2125-33.

12. Liu L, Zhang W, Qi X, et al. Randomized study of autologous cytokine-induced killer cell immunotherapy in metastatic renal carcinoma. Clin Cancer Res. 2012; 18:1751-9.

13. Pan K, Guan XX, Li YQ, et al. Clinical activity of adjuvant cytokine-induced killer cell immunotherapy in patients with post-mastectomy triple-negative breast cancer. Clin Cancer Res. 2014; 20:3003-11.

14. Pan K, Li YQ, Wang W, et al. The efficacy of cytokine-induced killer cell infusion as an adjuvant therapy for postoperative hepatocellular carcinoma patients. Ann Surg Oncol. 2013; 20:4305-11.

15. Pan QZ, Tang Y, Wang QJ, et al. Adjuvant cellular immunotherapy in patients with resected primary non-small cell lung cancer. Oncoimmunology. 2015; 4:e1038017.

16. Shi L, Zhou Q, Wu J, et al. Efficacy of adjuvant immunotherapy with cytokine-induced killer cells in patients with locally advanced gastric cancer. Cancer Immunol Immunother. 2012; 61:2251-9.

17. Wang Z, Zhang Y, Liu Y, et al. Association of myeloid-derived suppressor cells and efficacy of cytokine-induced killer cell immunotherapy in metastatic renal cell carcinoma patients. J Immunother. 2014; 37:43-50.

18. Weng DS, Zhou J, Zhou QM, et al. Minimally invasive treatment combined with cytokine-induced killer cells therapy lower the short-term recurrence rates of hepatocellular carcinomas. J Immunother. 2008; 31:63-71.

19. Kim JS, Kim YG, Pyo M, et al. Adoptive Cell Therapy of Melanoma with Cytokine-induced Killer Cells. Immune Netw. 2015; 15:58-65.

20. Hontscha C, Borck Y, Zhou H, et al. Clinical trials on CIK cells: first report of the international registry on CIK cells (IRCC). J Cancer Res Clin Oncol. 2011; 137:305-10.

21. Hui KM. CIK cells--current status, clinical perspectives and future prospects--the good news. Expert Opin Biol Ther. 2012; 12:659-61.

22. Ma Y, Zhang Z, Tang L, et al. Cytokine-induced killer cells in the treatment of patients with solid carcinomas: a systematic review and pooled analysis. Cytotherapy. 2012; 14:483-93.

23. Mesiano G, Todorovic M, Gammaitoni L, et al. Cytokine-induced killer (CIK) cells as feasible and effective adoptive immunotherapy for the treatment of solid tumors. Expert Opin Biol Ther. 2012; 12:673-84.

24. Thanendrarajan S, Kim Y, Schmidt-Wolf I. New adoptive immunotherapy strategies for solid tumours with CIK cells. Expert Opin Biol Ther. 2012; 12:565-72.

25. Li Y, Pan K, Liu LZ, et al. Sequential Cytokine-Induced Killer Cell Immunotherapy Enhances the Efficacy of the Gemcitabine Plus Cisplatin Chemotherapy Regimen for Metastatic Nasopharyngeal Carcinoma. PLoS One. 2015; 10:e0130620.

26. Li JJ, Gu MF, Pan K, et al. Autologous cytokine-induced killer cell transfusion in combination with gemcitabine plus cisplatin regimen chemotherapy for metastatic nasopharyngeal carcinoma. J Immunother. 2012; 35:189-95.

27. Hanahan D, Weinberg RA. Hallmarks of cancer: the next generation. Cell. 2011; 144:646-74

28. Yang L, Ren B, Li H, et al. Enhanced antitumor effects of DC-activated CIKs to chemotherapy treatment in a single cohort of advanced non-small-cell lung cancer patients. Cancer Immunol Immunother. 2013; 62:65-73.

29. Zhu Y, Zhang H, Li Y, et al. Efficacy of postoperative adjuvant transfusion of cytokine-induced killer cells combined with chemotherapy in patients with colorectal cancer. Cancer Immunol Immunother. 2013; 62:1629-35.

30. Schmidt-Wolf IG, Lefterova $P$, Johnston $V$, et al. Sensitivity of multidrug-resistant tumor cell lines to immunologic effector cells. Cell Immunol. 1996; 169:85-90.
31. Zhang YS, Yuan FJ, Jia GF, et al. CIK cells from patients with HCC possess strong cytotoxicity to multidrug-resistant cell line Bel-7402/R. World J Gastroenterol. 2005; 11:3339-45.

32. Yang L, Du C, Wu L, et al. Cytokine-Induced Killer Cells Modulates Resistance to Cisplatin in the A549/DDP Cell Line. J Cancer. 2017;8(16):3287-95.

33. Gammaitoni L, Giraudo L, Leuci V, et al. Effective activity of cytokine-induced killer cells against autologous metastatic melanoma including cells with stemness features. Clin Cancer Res. 2013; 19:4347-58.

34. Sangiolo D, Mesiano G, Gammaitoni L, et al. Cytokine-induced killer cells eradicate bone and soft-tissue sarcomas. Cancer Res. 2014; 74:119-29.

35. Wei F, Rong XX, Xie RY, et al. Cytokine-induced killer cells efficiently kill stem-like cancer cells of nasopharyngeal carcinoma via the NKG2D-ligands recognition. Oncotarget.2015; 6 (33): 35023-39.

36. Zitvogel L, Galluzzi L, Smyth MJ, et al. Mechanism of action of conventional and targeted anticancer therapies: reinstating immunosurveillance. Immunity. 2013; 39:74-88

37. Hoyle C, Bangs CD, Chang $\mathrm{P}$, et al. Expansion of Philadelphia chromosome-negative CD3(+)CD56(+) cytotoxic cells from chronic myeloid leukemia patients: in vitro and in vivo efficacy in severe combined immunodeficiency disease mice. Blood. 1998; 92:3318-27.

38. Shi M, Zhang B, Tang ZR, et al. Autologous cytokine-induced killer cell therapy in clinical trial phase I is safe in patients with primary hepatocellular carcinoma. World J Gastroenterol.2004; 10:1146-51.

39. Herber DL, Nagaraj S, Djeu JY, et al. Mechanism and therapeutic reversal of immune suppression in cancer. Cancer Res. 2007; 67:5067-9.

40. Comoli P. Adoptive transfer of allogeneic Epstein-Barr virus (EBV)-specific cytotoxic $T$ cells with in vitro antitumor activity boosts LMP2-specific immune response in a patient with EBV-related nasopharyngeal carcinoma. Annals of Oncology. 2004; 15:113-7.

41. Comoli P, Pedrazzoli P, Maccario R, et al. Cell therapy of stage IV nasopharyngeal carcinoma with autologous Epstein-Barr virus-targeted cytotoxic T lymphocytes. J Clin Oncol. 2005; 23:8942-9.

42. Chia WK, Teo M, Wang WW, et al. Adoptive T-cell transfer and chemotherapy in the first-line treatment of metastatic and/or locally recurrent nasopharyngeal carcinoma. Mol Ther. 2014; 22:132-9. 